\title{
Short-lived $p$-nuclides in the early solar system and implications on the nucleosynthetic role of X-ray binaries
}

\author{
N. Dauphas ${ }^{\mathrm{a}}$, T. Rauscher ${ }^{\mathrm{b}}$, B. Marty ${ }^{\mathrm{c}}$, and L. Reisberg ${ }^{\mathrm{c}}$ \\ ${ }^{a}$ Enrico Fermi Institute, The University of Chicago, 5640 South Ellis Avenue, Chicago \\ IL 60637, USA (dauphas@uchicago.edu)

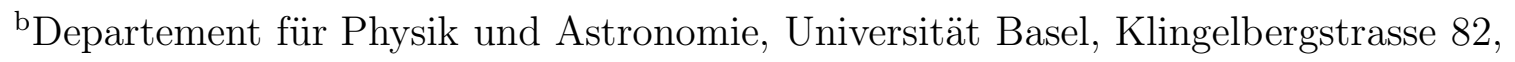 \\ CH-4056 Basel, Schweiz \\ ${ }^{\mathrm{c}}$ Centre de Recherches Pétrographiques et Géochimiques, CNRS UPR 2300, 15 rue \\ Notre-Dame des Pauvres, BP 20, 54501 Vandœuvre-lès-Nancy Cedex, France.
}

The data available for short-lived $p$-nuclides are used in an open nonlinear model of the chemical evolution of the Galaxy in order to discuss the origin of extinct radionuclides, the stellar sources of $p$-nuclides, and the chronology of solar system formation. It is concluded that the observed abundances of ${ }^{97} \mathrm{Tc},{ }^{98} \mathrm{Tc},{ }^{92} \mathrm{Nb}$, and ${ }^{146} \mathrm{Sm}$ in the early solar system are consistent with nucleosynthesis in type II supernovae during continuous chemical evolution of the Galaxy and a subsequent short isolation of the presolar molecular cloud from fresh nucleosynthetic inputs. However, further work on supernova models is needed before $p$-radionuclides will comprise reliable cosmochronometers. Despite these limitations, we argue that niobium-92 can be used to test whether the $r p$-process contributed to the synthesis of light $p$-nuclides in the Mo-Ru region.

\section{Introduction}

Extinct nuclides are radioactive nuclides that were present in the early solar system but have now decayed below detection levels. Their prior presence can be inferred from abundance variations of their daughter nuclides in solar system formation remnants. The origin of short-lived nuclides in the early solar system is the subject of much debate. They may have resulted from $(i)$ inheritence from the chemical evolution of the Galaxy [ 1, 2], (ii) injection from a nearby giant star that might have triggered the protosolar nebula into collapse [3], and (iii) irradiation within the solar system by intense stellar flares [ [, 5, 6, 7, 8]. In order to investigate the formation of the solar system, one can proceed either forwards or backwards in time. The abundances of short-lived $p$-nuclides in the interstellar medium at solar system birth can be derived from modeling of galactic chemical evolution [1], 2] and stellar nucleosynthesis [9, 10]. The abundances of short-lived $p$-nuclides in the early solar system can also be derived from laboratory measurements of solar system formation remnants. Comparison between the predicted and the observed abundances provides unequalled information on the origin of extinct radionuclides and solar system birth. 
Table 1

Abundances of short-lived $p$-nuclides. $\mathrm{R}$ is the initial ratio in the solar system derived from meteorite measurements, $\mathrm{P}$ is the production ratio in supernovae, $\Re_{\mathrm{ESS}}$ is the remainder ratio in the early solar system calculated as $\mathrm{R} / \mathrm{P}$, and $\Re_{\mathrm{ISM}}$ is the remainder ratio in the interstellar medium derived from GCE modeling (see text for details and references).

\begin{tabular}{lccccc}
\hline \hline Nuclide & & $\mathrm{R}$ & $\mathrm{P}$ & $\Re_{\mathrm{ESS}}$ & $\Re_{\mathrm{ISM}}$ \\
\hline Technetium-97 & ${ }^{97} \mathrm{Tc} /{ }^{98} \mathrm{Ru}$ & $<4 \times 10^{-4}$ & $4.4 \pm 1.3 \times 10^{-2}$ & $<1.3 \times 10^{-2}$ & $1.2 \pm 0.3 \times 10^{-3}$ \\
Technetium-98 & ${ }^{98} \mathrm{Tc} /{ }^{8} \mathrm{Ru}$ & $<8 \times 10^{-5}$ & $7.3 \pm 2.5 \times 10^{-3}$ & $<1.7 \times 10^{-2}$ & $1.9 \pm 0.5 \times 10^{-3}$ \\
Niobium-92 & ${ }^{92} \mathrm{Nb} /{ }^{92} \mathrm{Mo}$ & $2.8 \pm 0.5 \times 10^{-5}$ & $1.5 \pm 0.6 \times 10^{-3}$ & $1.9 \pm 0.8 \times 10^{-2}$ & $1.6 \pm 0.4 \times 10^{-2}$ \\
Samarium-146 & ${ }^{146} \mathrm{Sm} /{ }^{144} \mathrm{Sm}$ & $7.6 \pm 1.3 \times 10^{-3}$ & $1.8 \pm 0.6 \times 10^{-1}$ & $4.2 \pm 1.6 \times 10^{-2}$ & $4.6 \pm 1.1 \times 10^{-2}$ \\
\hline
\end{tabular}

\section{Abundances in the early solar system}

Four short-lived $p$-nuclides might have been alive in the early solar system; ${ }^{97} \mathrm{Tc}$ which EC-decays to ${ }^{97} \mathrm{Mo}$ (mean life of $3.8 \mathrm{Ma}$ ), ${ }^{98} \mathrm{Tc}$ which $\beta$-decays to ${ }^{98} \mathrm{Ru}(6.1 \mathrm{Ma}),{ }^{92} \mathrm{Nb}$ which EC-decays to ${ }^{92} \mathrm{Zr}$ (50.1 Ma), and ${ }^{146} \mathrm{Sm}$ which $\alpha$-decays to ${ }^{142} \mathrm{Nd}$ (149 Ma). Among these, only upper limits could be derived for ${ }^{97} \mathrm{Tc}$ [ 11, 12] and ${ }^{98} \mathrm{Tc}$ [ 13], while the abundances of ${ }^{92} \mathrm{Nb}$ [ 14, 15] and ${ }^{146} \mathrm{Sm}$ [ 16, 17, 18] are firmly established. The initial abundances normalized to a stable neighbor nuclide synthesized by the same process are compiled in Table 1.

\section{Stellar production ratios}

The origin of $p$-nuclides still poses some puzzles. The currently most favored production process is by photodisintegration of seed nuclides, this is called the $\gamma$-process. However, as discussed below, there still remains the problem of finding the proper site for the $\gamma$ process. Furthermore, it is unknown whether the $\gamma$-process is the only type of process that contributed to $p$-nuclide production. In the following we discuss two production sites, type II and type Ia supernovae. The feasibility of an additional scenario, the $r p$-process, will be discussed in Sec. 7 .

\subsection{Type II supernovae}

Currently, core-collapse supernovae of type II (SNII) are strongly favored to be the site for the so-called $p$ - or $\gamma$-process outlined above. The necessary temperatures of about $2 \leq T_{9} \leq 3$ can be reached during hydrostatic and explosive neon/oxygen-burning [ 19, 20, 21, 22, 10, 23, 24, 25]. However, modern models still fail to reproduce the full range of $p$-nuclides although they succeed in producing substantial amounts in several mass ranges. More pronounced is the underproduction in the Mo-Ru region. For the initial production ratios ejected from the site of the $p$-process and used in our chemical evolution model described in Sec. 4, we have taken ratios from a recent study of nucleosynthesis in SNII [26]. While $p$-nuclides with $A>100-110$ are produced in amounts in rough agreement with solar abundances, for the Mo-Ru region the new model also exhibits underproduction features similar to those found in previous studies [ [19, [10]. The reasons for this remain unclear. Details of the production are strongly dependent on stellar physics, such as the convection treatment used. Furthermore, the majority of the photodisintegration rates acting in this region are derived from theory. However, the experimental neutron capture data [27] as well as the rare data on proton capture in this region [28] agree well 
with the predictions [29, 30]. Additionally, an increased amount of seed nuclei would be needed in order to account for the missing abundances [19]. For our purposes, the absolute production factors are not important as we use abundance ratios. Obviously, in the following we have to assume that those ratios will not change when the reason for the underproduction is identified. We used the SNII yields for three progenitor masses, 15, 21, and $25 \mathrm{M}_{\odot}$. These yields were integrated over the initial mass function IMF, describing the birth rate $\phi$ of stars with different initial mass $m(\phi \propto \mathrm{d} N / \mathrm{d} m)$. For stars with initial mass higher than $\mathrm{M}_{\odot}$, the initial mass function follows a power law of the mass $\phi \propto m^{-\alpha}$, where $\alpha=2.3 \pm 0.7$ [31]. In order to calculate the IMF-averaged yields, the mass interval 12.5-27.5 was further divided into three intervals $12.5-17.5,17.5-22.5$, and $22.5-27.5 \mathrm{M}_{\odot}$. The yields of the 15,21 , and $25 \mathrm{M}_{\odot}$ mass stars were then weighted by the IMF integrated over the three mass intervals. The IMF-integrated SNII production ratios are compiled in Table 1.

\subsection{Type Ia supernovae}

Additional sites for the $p$-process have mainly drawn interest because of the problem with producing the Mo-Ru region. It has been proposed that the temperatures required for the photodisintegrations of the $p$-process could be reached in the C-rich zone of type Ia supernovae (SNIa) [9]. Early claims that the problem in the Mo-Ru region could also be alleviated have not been substantiated by making use of more realistic astrophysical models [ 32, 10]. Howard and Meyer [32] find that they cannot produce $p$-nuclei in sufficient quantities to make a significant galactic contribution unless the $s$-process seeds are enhanced by several orders of magnitude. Furthermore, they observe that the production trends with respect to mass number are similar to those observed in SNII models. This can be understood by the fact that the burning during accretion on the $\mathrm{C} / \mathrm{O}$ core proceeds in a similar manner as the late burning stages of SNII progenitors. The actual photodisintegration process is the same. The above results seem to support the view that either SNIa do not contribute to the $p$-ratios or, if they do, the ratios are not significantly changed from those obtained with SNII models.

\section{Open nonlinear galactic chemical evolution}

The chemical evolution of the Galaxy in the solar neighborhood is governed by several mechanisms [2, 33, 34]. We assume that the surface density of the galactic disk in the solar neighborhood $\Sigma$ grew with time from zero to its present-day value as a result of the accretion of low-metallicity gas. The infall rate is modelled as a normal density law $\mathcal{G}$ with standard deviation equal to the mean $\tau$, where $\tau$ is the infall time-scale [35],

$\frac{\mathrm{d} \Sigma}{\mathrm{d} t}=\xi \mathcal{G}(\tau)$

The net evolution of the gas surface density $\Sigma_{\mathrm{g}}$ is a balance between star formation and disruption on one hand, and infall of low metallicity gas on the other hand. The rate of star formation depends on the gas density $\left(\Sigma_{g}\right)$ according to Schmidt's law,

$\frac{\mathrm{d} \Sigma_{\mathrm{g}}}{\mathrm{d} t}=-\omega \Sigma_{\mathrm{g}}^{\mathrm{n}}+\xi \mathcal{G}(\tau)$ 
For a nuclide for which the instantaneous recycling approximation is valid, its concentration in the interstellar medium $\mathrm{Z}$ evolves through time as a result of stellar nucleosynthesis ( $\mathrm{y}$ is the yield defined as the ratio of newly created matter of that nucleus to newly created mass of permanent stellar remnants), dilution by infall of low-metallicity gas, and radioactive decay,

$\frac{\mathrm{dZ}}{\mathrm{d} t}=\mathrm{y} \omega \Sigma_{\mathrm{g}}^{\mathrm{n}-1}-\frac{\xi \mathcal{G}(\tau)}{\Sigma_{\mathrm{g}}}\left(\mathrm{Z}-\mathrm{Z}_{\xi}\right)-\lambda \mathrm{Z}$

Three parameters $(\xi, \tau$, and $\omega)$ are enough to describe the chemical evolution of the Galaxy in the solar neighborhood and to characterize its most important features. In order to constrain these parameters, we proceeded like Sommer-Larsen [36]. The present disk surface density, gas mass fraction, and metallicity in the solar neighborhood are known. Knowledge of these boundary conditions allows a straightforward derivation of the dependence of $\xi, \omega$, and $\mathrm{y}_{\mathrm{o}}$ upon $\tau$. The G-dwarf metallicity distribution is used in order to estimate $\tau$ and all other parameters which depend on it using a minimization algorithm.

The exponent of the Schmidt power law is estimated to be $1.4 \pm 0.15$ [ 37, 38]. The initial disk density is taken to be zero and its present-day value $45 \pm 5 \mathrm{M}_{\odot} \mathrm{pc}^{-2}$ [39, 40]. The gas density is estimated to be $13 \pm 4 \mathrm{M}_{\odot} \mathrm{pc}^{-2}$ [39]. The metallicity at solar system birth is $[\mathrm{O} / \mathrm{H}]=0$. The metallicity of the infalling gas is assumed to have been constant through time, $[\mathrm{O} / \mathrm{H}]=-1$ [ 36, 41]. The age of the Galaxy is estimated to be $13.2 \pm 1.5$ $\mathrm{Ga}$ [ 42]. The G-dwarf metallicity distribution in the solar neighborhood is taken from Haywood [ 43]. The model that best fits the G-dwarf metallicity distribution (Fig. 1) corresponds to $(\xi, \tau, \omega)=(56,4.8,0.065)$.

Simulations provide a reliable means of estimating uncertainties on model parameters. Several sets of possible observations are first simulated. Then, each simulated set is used to compute a set of possible model parameters. Finally, this set of possible model parameters is examined for its distribution. The exponent of the Schmidt power law, the present surface density of the Galaxy in the solar neighborhood, and the gas density were simulated as normal density laws. Residuals between the best fit G-dwarf metallicity distribution and the observed distribution were calculated. These residuals were then resampled with repetition and added to the best fit distribution. This bootstrap procedure permitted the simulation of a set of possible G-dwarf metallicity distributions. Using the fitted parameters, it is then possible to calculate the theoretical abundance of a $p$-nuclide in the interstellar medium at the time of solar system birth and its associated uncertainty.

\section{The remainder ratio}

The remainder ratio $(\Re)$ is defined as the ratio of the abundance of a radioactive nuclide to the abundance it would have if it were stable [ 33]. The remainder ratio in the interstellar medium at the time of isolation of the presolar molecular cloud from fresh nucleosynthetic inputs $\Re_{\text {ISM }}$ can be evaluated within the framework of the galactic chemical evolution model that we have presented,

$\Re_{\mathrm{ISM}}=\int_{T_{\odot}} \sum_{\mathrm{g}}^{\mathrm{n}-1} \mathrm{e}^{\theta+\lambda t} \mathrm{~d} t / \int_{T_{\odot}} \sum_{\mathrm{g}}^{\mathrm{n}-1} \mathrm{e}^{\theta+\lambda T_{\odot}} \mathrm{d} t$, 


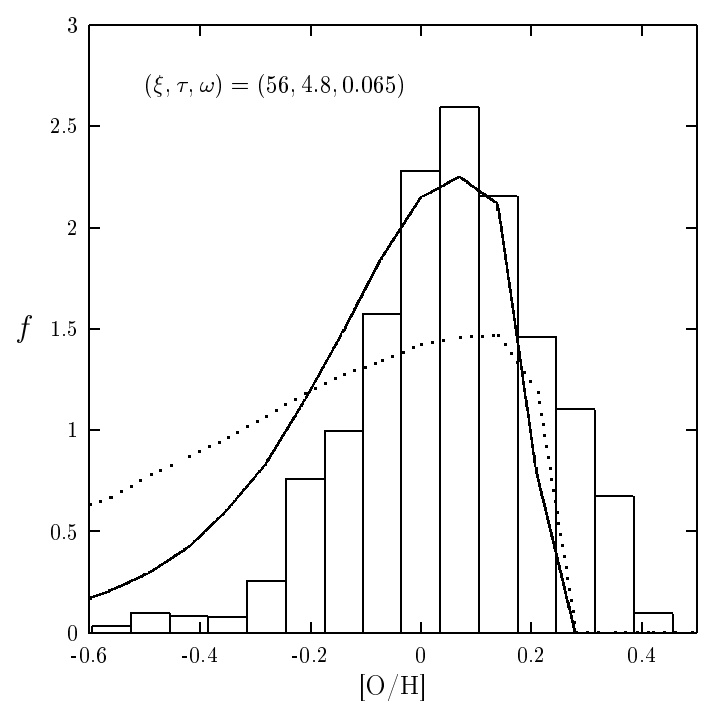

Figure 1. G-dwarf metallicity distribution (density) in the solar neighborhood [ 43]. The $[\mathrm{Fe} / \mathrm{H}]$ was converted to $[\mathrm{O} / \mathrm{H}]$ using $[\mathrm{O} / \mathrm{H}]=0.70 \pm 0.04[\mathrm{Fe} / \mathrm{H}]+0.07 \pm 0.02$ [ 50]. Histogram: observations [ 43], dotted line: closed-box model, solid line: best infall model. The parameters describing the best infall model are indicated at the top left corner.

where $\theta$ is the cycle number defined as $\mathrm{d} \theta / \mathrm{d} t=\xi \mathcal{G}(\tau) / \Sigma_{\mathrm{g}}$ [ [33]. Using the parameters calculated in the previous section, it can be shown that for short-lived nuclides, the remainder ratio takes the form,

$\Re_{\mathrm{ISM}}=\kappa /\left(\lambda \mathrm{T}_{\odot}\right)$,

with $\kappa=2.7 \pm 0.4$, where the confidence interval accounts for model uncertainties and $\mathrm{T}_{\odot}$ is the presolar age of the Galaxy $8.7 \pm 1.5 \mathrm{Ga}, \kappa=1$ corresponds to the closed-box model. Clayton [2] estimated within a linear model that $\kappa$ must be within 2 and 4 . The remainder ratio within the solar system at solar system birth $\Re_{\mathrm{ESS}}$ can also be estimated using stellar production ratios and observed abundances in meteorites,

$\Re_{\mathrm{ESS}}=\mathrm{R} / \mathrm{P}$,

where $\mathrm{R}$ is the early solar system ratio of a radioactive nuclide to a stable nuclide produced by the same process and $\mathrm{P}$ is the production ratio. There might have been a delay between isolation of the presolar molecular cloud from fresh nucleosynthetic inputs and solar system formation,

$\Re_{\mathrm{ESS}}=\Re_{\mathrm{ISM}} \mathrm{e}^{-\lambda \Delta}$,

where $\lambda$ is the decay constant and $\Delta$ is the free decay interval. If all short-lived $p$-nuclides were derived from the chemical evolution of the Galaxy, they should define a unique free decay interval. It is worthwhile to note that the deterministic chemical evolution model of the Galaxy probably breaks down for very short-lived nuclides because granularity might 


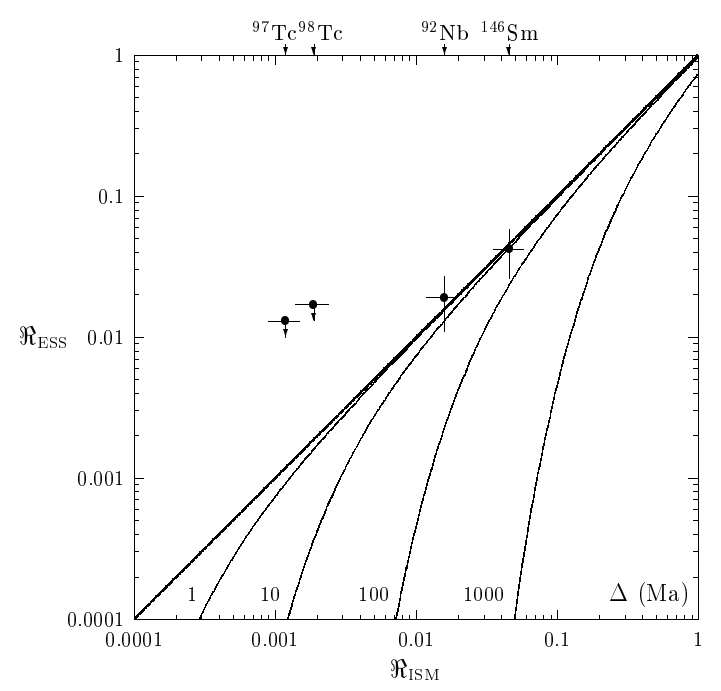

Figure 2. The remainder ratio in the early solar system $\Re_{E S S}$ is shown as a function of the remainder ratio in the interstellar medium at solar system birth $\Re_{\mathrm{ISM}}$ for a variety of free decay intervals $\Delta$. The points represent the positions of short-lived $p$-nuclides in this diagram (Table 1, see text for details).

play a role, which would require a stochastic treatment [44]. The remainder ratios of ${ }^{97} \mathrm{Tc},{ }^{98} \mathrm{Tc},{ }^{92} \mathrm{Nb}$, and ${ }^{146} \mathrm{Sm}$ are compiled in Table 1 . As can be seen in Fig. 2, they are consistent with synthesis of short-lived $p$-nuclides in supernovae and derivation from the interstellar medium with a comparatively short free decay interval $(\Delta<100 \mathrm{Ma})$.

\section{Origin of extinct radioactivities}

Assuming that there was no free decay interval between isolation of the presolar molecular cloud and condensation of the first solids in the solar system, our galactic chemical evolution model predicts that the initial ${ }^{97} \mathrm{Tc} /{ }^{98} \mathrm{Ru}$ and ${ }^{98} \mathrm{Tc} /{ }^{98} \mathrm{Ru}$ ratios at solar system birth were $5.3 \pm 2.0 \times 10^{-5}$ and $1.4 \pm 0.6 \times 10^{-5}$, respectively. These values are consistent with the upper-limits derived from measurements (Sec. 1).

There are a few very short-lived nuclides that cannot easily be explained by long-term chemical evolution of the Galaxy. These short-lived nuclides are ${ }^{7} \mathrm{Be},{ }^{10} \mathrm{Be},{ }^{26} \mathrm{Al},{ }^{53} \mathrm{Mn}$, and ${ }^{41} \mathrm{Ca}$. They may have been injected in the nascent solar system by a nearby giant star that might have triggered the protosolar cloud into collapse [3]. They may also have been synthesized within the solar system by local irradiation [ 4, 5, 6, 7, 8]. Both models face difficulties but the recent discovery of ${ }^{10} \mathrm{Be}$ [ 5, 45] and possibly ${ }^{7} \mathrm{Be}$ [7] in refractory inclusions favors the local irradiation model [4, 5, 6, 7, 8].

\section{Niobium-92 and $r p$-nucleosynthesis in X-ray binaries}

As discussed previously, supernovae models fail to reproduce the absolute abundance of $p$-nuclides in the Mo- $\mathrm{Ru}$ region. This failure may be due to an inadequacy of the stellar models, involved nuclear physics, or might indicate that other stellar sources contributed 
to the nucleosynthesis of $p$-nuclides. Interestingly, niobium-92 is a $p$-nuclide located in the mass range that shows a severe underproduction feature. Thus, this nuclide may provide useful constraints on $p$-nucleosynthesis. As indicated by other extinct $r$ and $p$ nuclides, it seems that the free decay interval was short, probably lower than a few hundreds of million years. Knowing the free decay interval, it is possible to do the reverse calculation and to compute the stellar ${ }^{92} \mathrm{Nb} /{ }^{92}$ Mo production ratio using the observed abundance in the early solar system and the galactic chemical evolution model presented previously. We thus estimate that the stellar ${ }^{92} \mathrm{Nb} /{ }^{92}$ Mo ratio was necessarily higher than $1.7 \pm 0.5 \times 10^{-3}$ $(\Delta>0)$ and was probably lower than $0.1(\Delta<200 \mathrm{Ma})$.

Another means of producing proton-rich nuclei is the $r p$-process [46]. In X-ray binaries, a thermonuclear runaway is ignited in the proton-rich layer accreted on the surface of a neutron star by mass flow from a companion star [47, 48]. It has been shown that proton-rich nuclei can be synthesized in the subsequent explosive $\mathrm{H}$ and He burning. The $r p$-process path closely follows the proton drip-line. In the final freeze-out phase, the nuclides in the $r p$-path decay towards stability and form $p$-nuclides [46]. Recently, a definite endpoint of the $r p$-process was identified which confines the possible production of $p$-nuclides to the mass region of about $A<110$ [49]. Thus, this scenario would not interfere with the production in SNII but rather might account for the missing abundances in the Mo-Ru region. Among the isotopes considered here, the $r p$-process could contribute to the abundances of ${ }^{92} \mathrm{Mo},{ }^{97} \mathrm{Tc}$, and ${ }^{98} \mathrm{Ru}$. The nuclides ${ }^{92} \mathrm{Nb}$ and ${ }^{98} \mathrm{Tc}$ cannot be synthesized because they are shielded by stable nuclides.

Niobium-92 is the only $p$-nuclide in the Mo-Ru region that cannot be synthetized in $\mathrm{X}$-ray bursts but was alive in the early solar system. Thus, the abundance of this nuclide in the solar system may be used to test whether X-ray bursts significantly contributed to the synthesis of proton-rich nuclides. If X-ray bursts produced $90 \%$ of the $p$-nuclides in the Mo-Ru region and supernovae synthesized the rest, then the ${ }^{92} \mathrm{Nb} /{ }^{92}$ Mo ratio expelled from stars to the interstellar medium would have been lowered by a factor of 10 relative to supernovae because X-ray bursts produce no ${ }^{92} \mathrm{Nb}$. Thus, the apparent production ratio would have been $1.5 \pm 1.0 \times 10^{-4}$, a factor of 10 lower than the required minimum production ratio of $1.7 \pm 0.5 \times 10^{-3}$. This result speaks against a significant contribution of X-ray binaries to the synthesis of $p$-nuclides in the Mo- $\mathrm{Ru}$ region. Note that if $\mathrm{X}$ ray binaries contributed to the synthesis of $p$-nuclides in the Mo-Ru region, they would overproduce nuclides in the $A=104$ region [49]. The problem of the origin of the Mo and $\mathrm{Ru} p$-nuclei therefore remains unsolved and clearly warrants further research.

We thank F.-K. Thielemann, H. Schatz, A.M. Davis, and G.W. Lugmair for extended discussions. This work is supported in part by the French CNES/INSU (PNP), the NASA (grant NAGW-9510), the Swiss NSF (grant 2000-061031.02), and the European Union (grant SMT4-CT98-2220). T. R. acknowledges support by a PROFIL professorship (Swiss NSF grant 2024-067428.01).

\section{REFERENCES}

1. D.N. Schramm, G.J. Wasserburg, Astrophys. J. 162 (1970) 57.

2. D.D.Clayton, in: W.D. Arnett, J.W. Truran (Eds.), Nucleosynthesis: Challenges \& New Developments, University of Chicago Press, Chicago, 1985, p. 65.

3. A.G.W. Cameron, H. Vanhala, P. Höflich, in: T.J. Bernatowicz, E. Zinner (Eds.), Astrophysical Im- 
plications of the Laboratory Study of Presolar Materials, American Institute of Physics, Woodbury, 1997, p. 665.

4. T. Lee, F.H. Shu, H. Shang, A.E. Glassgold, K.E. Rehm, Astrophys. J. 506 (1998) 898.

5. K.D. McKeegan, M. Chaussidon, F. Robert, Science 289 (2000) 1334.

6. M. Gounelle, F.H. Shu, H. Shang, A.E. Glassgold, K.E. Rehm, T. Lee, Astrophys. J. 548 (2001) 1051.

7. M. Chaussidon, F. Robert, K.D. McKeegan, Lunar Planet. Sci. XXXIII (2002) \#1563.

8. I. Leya, R. Wieler, A.N. Halliday, Lunar Planet. Sci. XXXIII (2002) \#1268.

9. W.M. Howard, B.S. Meyer, S.E. Woosley, Astrophys. J. Lett. 373 (1991) L5.

10. M. Rayet, M. Arnould, M. Hashimoto, N. Prantzos, K. Nomoto, Astron. Astrophys. 298 (1995) 517.

11. N. Dauphas, B. Marty, L. Reisberg, Astrophys. J. 565 (2002) 640.

12. N. Dauphas, B. Marty, L. Reisberg, Astrophys. J. Lett. 569 (2002) L139.

13. H. Becker, R.J. Walker, Eleventh Annual V.M. Goldschmidt Conference (2001) \#3047.

14. C.L. Harper Jr., Astrophys. J. 466 (1996) 437.

15. M. Schönbächler, et al., Science 295 (2002) 1705.

16. G.W. Lugmair, S.J.G. Galer, Geochim. Cosmochim. Acta 56 (1992) 1673.

17. A. Prinzhofer, D.A. Papanastassiou, G.J. Wasserburg, Geochim. Cosmochim. Acta 56 (1992) 797.

18. L.E. Nyquist, B. Bansal, H. Wiesmann, C.-Y.Shih, Meteoritics 29 (1994) 872.

19. S.E. Woosley, W.M. Howard, Astrophys. J. Suppl. S. 36 (1978) 285.

20. N. Prantzos, M. Hashimoto, M. Rayet, M. Arnould, Astron. Astrophys. 238 (1990) 455.

21. M. Rayet, N. Prantzos, M. Arnould, Astron. Astrophys. 227 (1990) 271.

22. D.L. Lambert, Astron. Astrophys. Rev. 3 (1992) 201.

23. G. Wallerstein, et al., Rev. Mod. Phys. 69 (1997) 995.

24. M. Arnould, K. Takahashi, Rep. Prog. Phys. 62 (1999) 395.

25. K.-H. Langanke, Nucl. Phys. A 654 (1999) 330.

26. T. Rauscher, A. Heger, R.D. Hoffman, S.E. Woosley, Astrophys. J. 576 (2002) 323.

27. Z.Y. Bao, H. Beer, F. Käppeler, F. Voss, K. Wisshak, T. Rauscher, At. Data Nucl. Data Tables 76 (2000) 70 .

28. S. Harissopulos, et al., Phys. Rev. C 64 (2001) 055804.

29. T. Rauscher, F.-K. Thielemann, At. Data Nucl. Data Tables 75 (2000) 1.

30. T. Rauscher, F.-K. Thielemann, At. Data Nucl. Data Tables 79 (2001) 47.

31. P. Kroupa, Mon. Not. R. Astron. Soc. 322 (2001) 231.

32. W.M. Howard, B.S. Meyer, in: F. Käppeler, K. Wisshak (Eds.), Nuclei in the Cosmos, IOP Publishing, Bristol, 1993, p. 575.

33. D.D. Clayton, Mon. Not. R. Astron. Soc. 234 (1988) 1.

34. B.E.J. Pagel, Nucleosynthesis \& Chemical Evolution of Galaxies, Cambridge University Press, Cambridge, 1997.

35. R.X. Chang R.X., J.L. Hou, C.G. Shu, C.Q. Fu, Astron. Astrophys. 350 (1999) 38.

36. J. Sommer-Larsen, Mon. Not. R. Astron. Soc. 249 (1991) 368.

37. J.P.E. Gerritsen, V. Icke, Astron. Astrophys. 325 (1997) 972.

38. R.C. Kennicutt Jr., Astrophys. J. 498 (1998) 541.

39. J. Holmberg, C. Flynn, Mon. Not. R. Astron. Soc. 313 (2000) 209.

40. M. Crézé, E. Chereul, O. Bienaymé, C. Pichon, Astron. Astrophys. 329 (1998) 920.

41. B.P. Wakker, et al., Nature 402 (1999) 388.

42. B. Chaboyer, in: T.V. Hippel, C. Simpson, N. Manset (Eds.), Astrophysical Ages and Time Scales, ASP Conference Series 245, Astronomical Society of the Pacific, San Francisco, 2001, p. 162.

43. M. Haywood, Mon. Not. R. Astron. Soc. 325 (2001) 1365.

44. B.S. Meyer, N. Luo, Lunar Planet. Sci. XXVIII (1997) \# 1841.

45. N. Sugiura, Y. Shuzou, A.A. Ulyanov, Meteoritics Planet. Sci. 36 (2001) 1397.

46. H. Schatz, et al., Phys. Rep. 294 (1998) 167.

47. R.K. Wallace, S.E. Woosley, Astrophys. J. Suppl. S. 45 (1981) 389.

48. R.E. Taam, S.E. Woosley, T.A. Weaver, D.Q. Lamb, Astrophys. J. 413 (1993) 324.

49. H. Schatz, et al., Phys. Rev. Lett. 86 (2001) 3471.

50. Y.Q. Chen, P.E. Nissen, G. Zhao, H.W. Zhang, T. Benoni, Astrophys. J. Suppl. S. 141 (2000) 491. 